the baseline $\mathrm{ADR}<25 \%(\mathrm{OR}=1.55,95 \% \mathrm{CI}=1.16-2.07$, $\mathrm{P}=0.003)$. Moreover, there was a significant difference in the right-side ADR $(p=0.002)$ and borderline statistical significance in the left-side ADR $(p=0.04)$ between the two groups. In addition, adding simethicone to the PEG solution improved bowel preparation and patient acceptability. (figure 1a)

Conclusions In summary, we believe that the addition of simethicone to PEG is a promising bowel preparation method for colonoscopy in clinical practice among Asian populations. Additional large clinical trials are necessary to validate our results.

\section{IDDF2019-ABS-0038 PET-CT ENTEROGRAPHY CAN HELP IN PREDICTING A CLINICALLY SIGNIFICANT DIAGNOSIS IN PATIENTS WITH ILEO-CECAL THICKENING}

Abhi Kumar Singh*, Pankaj Gupta, Rajender Kumar Basher, Kaushal Kishor Prasad, Harshal Surendra Mandavdhare, Usha Dutta, Vishal Sharma. Postgraduate Institute of Medical Education and Research, India

\subsection{6/gutjnl-2019-IDDFabstracts.123}

Background Ileo-cecal thickening is a common, nonspecific imaging finding with often uncertain significance. Role of Positron emission tomography with $18 \mathrm{~F}$-fluorodeoxyglucose integrated with computed tomographic enterography (FDG-PETCTE) in patients with ileo-cecal thickening is unclear.

Methods Consecutive patients with ileo-cecal thickening on USG from July 2017 to October 2018 underwent FDG-PETCTE followed by colonoscopy. They were divided in to two groups-those with clinically significant diagnosis (intestinal tuberculosis, Crohn's disease, infectious colitis, malignancy) and those with clinically insignificant diagnosis (normal colonoscopy or nonspecific terminal ileitis); Standardized uptake value (SUV)-max values from the ileo-cecal area were compared with the final diagnosis and area under the receiver operating characteristic (AUROC) was made to determine the best sensitivity and specificity to predict a clinically significant diagnosis.

Results Thirty-four patients were finally included, 23(67.6\%) were males and the mean age was $40.44 \pm 15.40$ years. The clinically significant diagnosis was made in $27(79.4 \%)$ patients whereas $7(20.6 \%)$ patients had a clinically insignificant diagnosis. Presence of mural thickening on CTE was present in $27(79.4 \%)$ patients. The mean SUVmax for cecum was found to be $5.68 \pm 4.09$, that for the terminal ileum was $4.19 \pm 2.52$ and for ileo-cecal valve was 4.62 \pm 3.00 . The region with the highest SUVmax value was taken for the purpose of comparisons and labelled as SUVmax (ICT). The mean SUVmax (ICT) for patients with clinically significant diagnosis was $7.162 \pm 4.382$ while those with clinically insignificant diagnosis was $3.629 \pm 0.502(\mathrm{P}<0.001)$. The area under ROC curve of the PET SUVmax (ICT) values for prediction of clinically significant diagnosis was .741 (95\% CI, 0.581-0.901). The SUVmax (ICT) value of more than 4.50 had a sensitivity of $70.4 \%$ and specificity of $100 \%$ in predicting the presence of clinically significant diagnosis whereas the value of 3.64 had a sensitivity of $74.1 \%$ and a specificity of $57.1 \%$ for picking a clinically significant diagnosis.

Conclusions PET-CT enterography could be useful in predicting if ileo-cecal thickening is due to a clinically significant underlying diagnosis and guiding the need of colonoscopy.

\section{IDDF2019-ABS-0041 THE EFFECT OF BANANA FRUIT (MUSA SAPIENTUM) AS AN ADJUNCT TO OMEPRAZOLE IN THE MANAGEMENT OF UNINVESTIGATED DYSPEPSIA}

January Ramirez*. Quezon City General Hospital and Medical Center Department of Family Medicine and Community Health, Philippines

\subsection{6/gutjnl-2019-IDDFabstracts.124}

Background Efficacy, safety, and cost-effectiveness of banana fruit as an adjunct to Omeprazole in the management of Uninvestigated Dyspepsia, (UD.)

To determine the efficacy, safety, and cost-effectiveness of banana fruit as an adjunct to Omeprazole in the management of UD.

Methods This is a therapeutic experimental study on the effectiveness of banana fruit as an adjunct to Omeprazole in the management of Dyspepsia consulting at Quezon City General Hospital Out-Patient Clinic.

The study was conducted from December-February 2018. Out of 100 subjects, 81 were included in the study. Using the systemic sampling method, patients were assigned to either Group I-39 or Group II-42. Omeprazole $20 \mathrm{mg} / \mathrm{tab}$ once a day for 2 weeks was given to Group II, whereas, 1 piece of banana fruit 3 times a day was added to the same dose of Omeprazole in Group II for 2 weeks. Talley Dyspepsia Symptoms Questionnaire Mean Global Symptom Index Scoring was used to monitor improvement in the severity of dyspeptic symptoms.

Results There were 81 subjects who completed the study. The mean age of Group I 13 (33\%) belonged to 25-30 years old age bracket, most subjects in Group II are from the 19-24 years old age bracket, 12(29\%). Both groups had more female than male subjects, Group I 23(59\%), Group II 21(50\%). Epigastric Pain was the predominant complaint on both groups with a mean of 10.08 in Group I and mean of 9.23 in Group II. As to the resolution of symptoms, Group II subjects had their symptoms resolved by the 3rd and 7th day of follow-up regardless of the MGSI score as compared to Group I subjects whose symptoms on day 7th of follow-up. Treatment in both groups was proven effective based on the resolution of symptoms within the treatment period; however, there was a faster resolution of symptoms in Group II with a mean day of 3.35 compared to 4.23 of Group I. No adverse effects were observed in both groups.

Conclusions Banana fruit as an adjunct in the treatment of UD was proven to be effective, safe and cost-effective.

\section{IDDF2019-ABS-0043 COMPARISON OF TWO METHODS FOR ENDOSCOPIC FULL-THICKNESS RESECTION OF GASTROINTESTINAL LESIONS USING OTSC}

Wenhai Wang*, Peng Li, Ming Ji, Yongjun Wang, Shengtao Zhu, Lihua Liu, Shutian Zhang Department of Gastroenterology, Beijing Friendship Hospital, Capital Medical University, China

\subsection{6/gutjnl-2019-IDDFabstracts. 125}

Background The aim of this study was to compare and analyze the feasibility and safety of two methods of endoscopic full-thickness resection(EFTR) for the management of challenging epithelial and subepithelial neoplasms that are not amenable to resection techniques.

Methods This was a retrospective case series study of patients underwent two methods of EFTR, either of which was 
resection using ESD knives and post-resection closure with OTSC(Group 1), the other was closure with OTSC and secondary EFTR with snare(Group 2).

Results Of 11 patients, 6 cases were in Group 1 and 5 cases in Group 2. The mean time of EFTR procedure was $76.83 \pm$ 34.97 minutes in Group 1 which is significantly longer than that of Group $2(\mathrm{P}=0.0128)$. The mean time of OSTC closure and length of hospital stay of Group 1 were also longer compared to Group 2, but the difference was not significant. Complete resection (R0) and technical success rate of Group 1 and Group 2 were $83.3 \%$ and $100 \%(\mathrm{P}=0.338)$.VAS scores of Group 1 after operation and 24 hours are significantly higher than those of Group $2(\mathrm{P}=0.047$ and $\mathrm{P}=0.009$, respectively). In Group 1, One patient had delayed perforation which led to fever and pneumoperitoneum, and one patient developed abdominal pain.No complications associated with endoscopic procedure was observed in Group 2.

Conclusions EFTR of pre-resection closure is potentially faster and safer compared with the concept of applying closure after EFTR. Larger prospective controlled studies comparing these two techniques are warranted in the future.

\section{IDDF2019-ABS-0044 UTILITY OF INTESTINAL TISSUE BIOPSY XPERT-MTB/RIF IN EVALUATION OF PATIENTS WITH ILEO-CAECAL ULCERS}

Balaji L Bellam*, Harshal Mandavdhare, Kusum Sharma, Siddharth Shukla, Hariom Soni, Praveen Kumar, Harjeet Singh, Kaushal Prasad, Usha Dutta, Vishal Sharma. Postgraduate Institute of Medical Education and Research, India

\subsection{6/gutjnl-2019-IDDFabstracts.126}

Background Ileo-cecal ulcers could result from a number of conditions including intestinal tuberculosis and Crohn's disease. We report the use of GeneXpert Mtb/Rif in the diagnosis of ITB in patients with ileo-cecal ulcers.

Methods One year retrospective study involving patients with ileo-cecal ulcers who underwent GeneXpert Mtb/Rif from the intestinal biopsies taken from ulcers. The results of the test were compared with the final diagnosis. We divided the patients into two groups: those with a final diagnosis of ITB and those with a final diagnosis other than ITB (OTH).

Results Of the 40 patients with ileo-cecal ulcers included, 23 $(57.5 \%)$ were females and the mean age was $32.92 \pm 12.78$ years. Abdominal pain was present in $33(88.5 \%)$ patients and diarrhea in 12 (30\%). Out of 40 patients, 25 had ITB and 15 patients were in the OTH group. The sensitivity, specificity, negative predictive value, the positive predictive value and accuracy of GeneXpert-Mtb/Rif for diagnosis of ITB was $32 \%$ (CI: $14.95-53.50 \%), \quad 100 \% \quad(78.2-100), \quad 46.88 \% \quad(40.27-$ $53.59 \%), 100 \& 57.50$ (40.89-72.89)\% respectively.

Conclusions A positive GeneXpert-Mtb/Rif is helpful in the diagnosis of ITB but the sensitivity is low.

\section{IDDF2019-ABS-0053 COMPARISON OF OUTCOMES OF COLORECTAL POLYPECTOMY USING TRADITIONAL SNARE AND ROTARY SNARE: A PROSPECTIVE RANDOMIZED CONTROLLED TRIAL}

${ }^{1} \mathrm{Lu} \mathrm{Xu}{ }^{*},{ }^{1} Z$ hixing Zhang, ${ }^{1}$ Jiarong Xie, ${ }^{2}$ Lei Xu, ${ }^{2}$ Weihong Wang. 'Ningbo University, College of Medicine, Ningbo, China; ${ }^{2}$ Department of Gastroenterology, Ningbo First Hospital, Ningbo, China

\subsection{6/gutjnl-2019-IDDFabstracts.127}

Background The aim of the current study was to compare the efficacy and safety of polypectomy by using rotary snare vs. traditional snare during colonoscopy.

Methods A single-center randomized controlled trial, which included consecutive participants who were $\geq 18$ years old and detected with polyp(s) during routine colonoscopy between July and September 2018, was conducted. Participants with colorectal polyps were randomized to receive polypectomy using rotary snares or traditional snares. The primary outcome measure was the comparison of the average time of removing a polyp between those two groups. The secondary outcome measure was to compare the polyp resection time by using SMSA (Size, Morphology, Site, and Access) scores.

Results A total of two hundred participants were included in this study. Of them, 100 participants were randomly assigned to the rotary snare group (214 polyps) and the other 100 participants were randomly assigned to the traditional group (232 polyps). The mean resection time was significantly shorter in the rotary snare group than in the traditional snare group (24.41[SD:18.14]seconds vs. 29.53[SD:25.74]seconds, $P=0.021$ ). In the subgroup analysis, the resection time was also shorter in the rotary snare group than the traditional
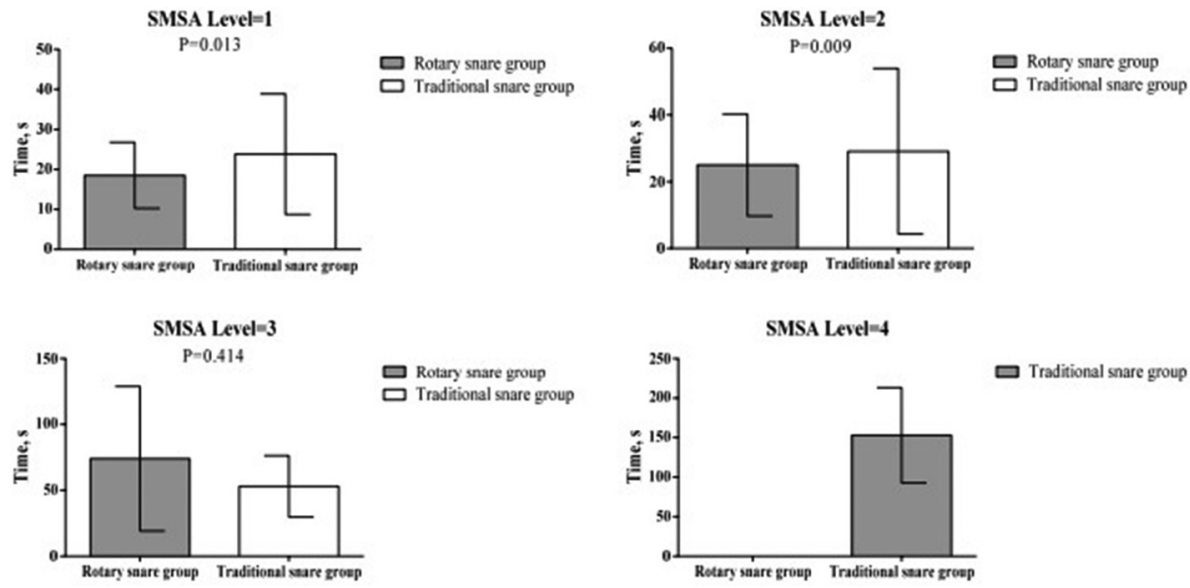

Abstract IDDF2019-ABS-0053 Figure 1 Comparison of polypectomy procedure times in size morphology site and access SMSA score between the traditional snare group and rotary snare group 